\title{
ORDER OF MAGNITUDE OF THE CONCENTRATION FUNCTION
}

\author{
PETER HALL
}

\begin{abstract}
Suppose a sum of independent random variables, when scaled in a suitable way, is stochastically compact. It is proved that the precise order of magnitude of the concentration function of the sum equals the inverse of the scale factor.
\end{abstract}

1. Introduction and results. Let $X, X_{1}, X_{2}, \ldots$ be independent and identically distributed random variables, and set $S_{n}=\Sigma_{1}^{n} X_{j}$. Weak limit theorems for $S_{n}$ usually take the form

$$
Y_{n} \equiv\left(S_{n}-\operatorname{med} S_{n}\right) / b_{n} \rightarrow Z
$$

in distribution, where med $S_{n}$ denotes a median of $S_{n},\left\{b_{n}\right\}$ is a sequence of norming constants and $Z$ is a nondegenerate limit. Feller [2] showed that interesting and useful results can still be obtained if the condition of convergence in distribution is weakened to that of stochastic compactness. The sequence $\left\{Y_{n}\right\}$ is stochastically compact if every subsequence contains a further subsequence converging in distribution to a proper, nondegenerate limit. We shall show in this paper that the condition of stochastic compactness permits a simple description of the precise order of magnitude of the concentration function.

The existence of a sequence $\left\{b_{n}\right\}$ such that $\left\{Y_{n}\right\}$ is stochastically compact, is equivalent to the condition

$$
\limsup _{x \rightarrow \infty} x^{2} P(|X|>x) / E\left\{X^{2} I(|X| \leqslant x)\right\}<\infty
$$

[2, p. 387]. Such constraints are related to characterisations of domains of partial attraction; see Jain and Orey [5] and Maller [6]. An alternative definition of stochastic compactness may be given in the following way. Define the function $v$ by

$$
\begin{aligned}
v(x) & =x^{-2} \int_{0}^{x} u P(|X|>u) d u \\
& =\frac{1}{2}\left[x^{-2} E\left\{X^{2} I(|X| \leqslant x)\right\}+P(|X|>x)\right], \quad x>0 .
\end{aligned}
$$

As was remarked in [4], $v$ is continuous, $v^{\prime}$ exists and is negative at continuity points of $|X|$, and $v$ is ultimately strictly decreasing. Hence the equation $v(\eta)=x^{-1}$ admits a unique solution, $\eta=\eta(x)$, for large $x$.

Received by the editors July 27, 1982 and, in revised form, October 25, 1982, January 19, 1983.

1980 Mathematics Subject Classification. Primary 60F99, 60G50; Secondary 60E99.

Key words and phrases. Concentration function, independent, median, norming constants, order of magnitude, sums of random variables. 
Lemma. Condition (1.1) is equivalent to the constraint that $\eta$ varies dominatedly at infinity. When either of these conditions holds and we take $b_{n}=\eta(n)$ in the definition of $Y_{n}$, the sequence $\left\{Y_{n}\right\}$ is stochastically compact.

The condition of dominated variation (equivalent here to $\mathrm{R}-\mathrm{O}$ variation) is discussed at length in the Appendix to Seneta [8]. In the present situation, dominated variation means that for some $\lambda>1, \eta(\lambda x) / \eta(x)$ is bounded as $x \rightarrow \infty$. We shall prove that when $\eta$ varies dominatedly, the sequence $\{1 / \eta(n)\}$ is of the same order of magnitude as the concentration function,

$$
Q\left(S_{n} ; h\right)=\sup _{x} P\left(x<S_{n} \leqslant x+h\right) .
$$

Therefore we may take $b_{n}=1 / Q\left(S_{n} ; h\right)$ in the definition of $Y_{n}$.

THEOREM. If $\eta$ varies dominatedly then for each $h>0$,

$$
0<\liminf _{n \rightarrow \infty} Q\left(S_{n} ; h\right) \eta(n) \leqslant \limsup _{n \rightarrow \infty} Q\left(S_{n} ; h\right) \eta(n)<\infty .
$$

This result greatly extends earlier estimates due to Esséen [1], who assumed $X$ to be in the domain of attraction of a stable law. See Petrov [7, Chapter III] for a review of the properties of concentration functions.

Since the preparation of this paper and its first revision, the author has seen a preprint by Griffin, Jain and Pruitt [3] which describes results which overlap with (but do not contain) those presented here.

\section{Proofs.}

Proof of Lemma. We prove first that the dominated variation of $\eta$ implies (1.1). If (1.1) fails then it fails along a subsequence $x_{k} \uparrow \infty$, which entails

$$
v\left(x_{k}\right) \sim \frac{1}{2} P\left(|X|>x_{k}\right)
$$

as $k \rightarrow \infty$. Whenever $0<\rho \leqslant 1$ we have

$$
\begin{aligned}
v(x) & \geqslant x^{-2} \int_{0}^{\rho x} u P(|X|>u) d u+x^{-2} P(|X|>x) \int_{\rho x}^{x} u d u \\
& =\rho^{2} v(\rho x)+\frac{1}{2}\left(1-\rho^{2}\right) P(|X|>x) .
\end{aligned}
$$

The right-hand side dominates $\frac{1}{2} P(|X|>x)$ (it is an increasing function of $\rho$, and so we let $\rho \rightarrow 0)$. Therefore it follows from (2.1) that $v\left(\rho x_{k}\right) \sim v\left(x_{k}\right)$ as $k \rightarrow \infty$. Let $\rho x_{k}=\eta\left(y_{k}\right)$, where $y_{k}$ depends on $\rho$, and observe that

$$
y_{k}^{-1}=v\left(\eta\left(y_{k}\right)\right)=v\left(\rho x_{k}\right) \sim v\left(x_{k}\right)=v\left(\rho^{-1} \eta\left(y_{k}\right)\right) .
$$

Suppose $\lambda>1$. If $\eta\left(\lambda y_{k}\right) \leqslant \rho^{-1} \eta\left(y_{k}\right)$ for all large $k$, then

$$
\left(\lambda y_{k}\right)^{-1}=v\left(\eta\left(\lambda y_{k}\right)\right) \geqslant v\left(\rho^{-1} \eta\left(y_{k}\right)\right) \sim y_{k}^{-1},
$$

which is impossible. Consequently $\limsup _{x \rightarrow \infty} \eta(\lambda x) / \eta(x) \geqslant \rho^{-1}$, and since this is true for all $\lambda>1$ and $0<\rho \leqslant 1, \eta$ cannot vary dominatedly. This contradiction proves (1.1).

Conversely, if (1.1) holds then the sequence $\left\{Y_{n}\right\}$ is stochastically compact whenever $b_{n} / a_{n}$ is bounded away from zero and infinity, where $a_{n}$ is given by

$$
n a_{n}^{-2} E\left\{X^{2} I\left(|X| \leqslant a_{n}\right)\right\}=1
$$


see [2, p. 387]. It is not difficult to show that for each $c>0, b_{n}=\eta(c n)$ satisfies this condition. (If $b_{n}$ satisfies the condition, so does $b_{n}^{\prime}=b_{[\mathrm{cn}]}$. It is readily checked that under (1.1), $b_{n}=\eta(n)$ satisfies the condition.) Therefore $\eta(c n) / \eta(n)$ is bounded away from zero and infinity as $n \rightarrow \infty$, for each $c>0$, and so (since $\eta$ is monotone) $\eta$ varies dominatedly.

Proof of Theorem. The symbol $C$ below denotes a generic positive constant. Let $\eta_{s}(n)$ ( $=\eta_{s}$ for short) and $v_{s}$ denote the versions of $\eta(n)$ and $v$ for the symmetrisation, $\tilde{X}$, of $X$. It was proved in [4] that $\eta(x) \leqslant \eta_{s}(2 x) \leqslant 2 \eta(4 x),{ }^{1}$ and from this it may be proved that $\eta$ varies dominatedly iff $\eta_{s}$ varies dominatedly.

Choose $n$ so large that $1-|\phi(t)|^{2} \leqslant \frac{1}{2}$ for $0 \leqslant t \leqslant 1 / \eta_{s}$, where $\phi$ denotes the characteristic function of $X$. By Esséen's [1] Main Lemma,

$$
\begin{aligned}
C Q\left(S_{n} ; h\right) & \geqslant \int_{0}^{1 / \eta_{s}}|\phi(t)|^{2 n} d t \geqslant \eta_{s}^{-1} \int_{0}^{1} \exp \left[-2 n E\left\{1-\cos \left(t \tilde{X} / \eta_{s}\right)\right\}\right] d t \\
& \geqslant \eta_{s}^{-1} \int_{0}^{1} \exp \left(-4 n\left[E\left\{\left(t \tilde{X} / \eta_{s}\right)^{2} I\left(|\tilde{X}| \leqslant \eta_{s}\right)\right\}+P\left(|\tilde{X}|>\eta_{s}\right)\right]\right) d t \\
& \geqslant \eta_{s}^{-1} \int_{0}^{1} \exp \left\{-8 n v_{s}\left(\eta_{s}\right)\right\} d t=\eta_{s}^{-1} e^{-8} .
\end{aligned}
$$

Since $\eta_{s}(n) \leqslant 2 \eta(2 n)$ then $Q\left(S_{n} ; h\right) \geqslant C / \eta(2 n)$, and the left-hand inequality in (1.2) follows from the dominated variation of $\eta$.

By the Main Lemma of [1] we have for each $\varepsilon>0$,

$$
\begin{aligned}
C(\varepsilon) Q\left(S_{n} ; h\right) & \leqslant \int_{0}^{\varepsilon}|\phi(t)|^{n} d t=\eta_{s}^{-1} \int_{0}^{\varepsilon \eta_{s}}\left|\phi\left(t / \eta_{s}\right)\right|^{n} d t \\
& \leqslant \eta_{s}^{-1} \int_{0}^{\varepsilon \eta_{s}} \exp \left[-(n / 2) E\left\{1-\cos \left(t \tilde{X} / \eta_{s}\right)\right\}\right] d t
\end{aligned}
$$

Since

$$
\begin{aligned}
E\{1-\cos (t \tilde{X})\} & \geqslant E\left[\{1-\cos (t \tilde{X})\} I\left(|\tilde{X}| \leqslant t^{-1}\right)\right] \\
& \geqslant C_{1} t^{2} E\left\{\tilde{X}^{2} I\left(|\tilde{X}| \leqslant t^{-1}\right)\right\} \geqslant C_{2} v_{s}\left(t^{-1}\right)
\end{aligned}
$$

for small $t$, using (1.1), then if $\varepsilon$ is sufficiently small,

$$
Q\left(S_{n} ; h\right) \leqslant C_{4} \eta_{s}^{-1} \int_{0}^{\varepsilon \eta_{s}} \exp \left\{-C_{3} n v_{s}\left(\eta_{s} / t\right)\right\} d t .
$$

Since $\eta_{s}$ is R-O varying, it may be deduced from Theorem A.1, p. 93 of [8] that for some $c \geqslant 1$ and $k \geqslant 1, \eta_{s}(x y) \leqslant \eta_{s}(x) y^{c}$ whenever $x \geqslant k$ and $y \geqslant k$. Therefore

$$
\eta_{s}(x) / t=\eta_{s}\left(x t^{-1 / c} t^{1 / c}\right) /\left(t^{1 / c}\right)^{c} \leqslant \eta_{s}\left(x t^{-1 / c}\right)
$$

and

$$
v_{s}\left(\eta_{s}(x) / t\right) \geqslant v_{s}\left(\eta_{s}\left(x t^{-1 / c}\right)\right)=t^{1 / c} / x,
$$

whenever $t \geqslant k^{c}$ and $x \geqslant k t^{1 / c}$. Thus, provided $n \geqslant k\left\{\varepsilon \eta_{s}(n)\right\}^{1 / c}$,

$$
n v_{s}\left(\eta_{s}(n) / t\right) \geqslant t^{1 / c}
$$

\footnotetext{
'Both inequalities are proved in [4], but only the first is used. In [4, p. 567, line 7], "first" should read "second".
} 
whenever $k^{c} \leqslant t \leqslant \varepsilon \eta_{s}(n)$. It also follows from Theorem A.l of [8] that for some $d>0, \eta_{s}(n) \leqslant n^{d}$ when $n$ is large. If we choose $c>d$ then (2.3) certainly holds for large $n$, and thus

$$
\int_{k^{c}}^{\varepsilon \eta_{s}} \exp \left\{-C_{3} n v_{s}\left(\eta_{s} / t\right)\right\} d t \leqslant \int_{0}^{\infty} \exp \left(-C_{3} t^{1 / c}\right) d t<\infty
$$

The right-hand inequality in (1.2) now follows from (2.2) and the fact that $\eta_{s}(n) \geqslant$ $\eta(n / 2)$.

ACKNOWLEDGEMENTS. I am grateful to the referee for his comments, particularly for pointing out the work in [2].

\section{REFERENCES}

1. C.-G. Esseen, On the concentration function of a sum of independent random variables, Z. Wahrsch. Verw. Gebiete 9 (1968), 290-308.

2. W. Feller, On regular variation and local limit theorems, Proc. Fifth Berkeley Sympos. Math. Statist. Prob. Vol. II, Contributions to Probability Theory, Part 1, Univ. of California Press, Berkeley. Calif., 1967, pp. 373-388.

3. P. S. Griffin, N. C. Jain and W. E. Pruitt, Approximate local limit theorems for laws outside domains of attraction, University of Minnesota Research Report 82-117.

4. P. Hall, Order of magnitude of moments of sums of random variables, J. London Math. Soc. 24 (1981), $562-568$.

5. N. C. Jain and S. Orey, Domains of partial attraction and tightness conditions, Ann. Probab. 8 (1980), 584-599.

6. R. A. Maller, A note on domains of partial attraction, Ann. Probab. 8 (1980), 576-583.

7. V. V. Petrov, Sums of independent random variables, Springer-Verlag, Berlin, Heidelberg and New York, 1975.

8. E. Seneta, Regularly varying functions. V, Lecture Notes in Math., vol. 508, Springer-Verlag, Berlin, Heidelberg and New York, 1976.

Department of Statistics, Faculty of Economics, Australian National University, P. O. Box 4, Canberra, ACT 2600, Australia 